\title{
Yeast Models for Amyloid Disorders
}

\author{
Y. Churnoff, M. Sun, H. Gong, G. Newnam, D. Kiktev, K. Bruce, R. Howie, T. Pan, A. Romanyuk
}

Georgia Institute of Technology, Atlanta GA

About 40 human diseases are associated with formation of the self-perpetuating fibrous cross- $\beta$ aggregates, termed amyloids. These include widespread Alzheimer's disease, associated with polymerization of amyloid beta (Abeta) peptide, and polyglutamine diseases (e. g. Huntington disease), associated with extensions of the polyQ stretches in certain proteins. Extreme case of amyloid disorders is represented by transmissible spongiform encephalopathies or prion diseases, caused by mammalian prion protein $(\mathrm{PrP})$ in an amyloidogenic (prion) form. Many amyloid diseases are fatal and incurable.

Therapeutic treatments typically only slow down the progression of the disease rather than addressing its cause, and prophylactic measures are lacking.

Age-dependence of some amyloidoses, such as Alzheimer's disease, will likely make them the most frequent cause of death with the further increase of the human life span. Mechanisms of amyloid formation and clearance are difficult to investigate in mammals due to long onset periods and lack of the reliable phenotypic assays. Yeast Saccharomyces cerevisiae contains several proteins with QN-rich domains, that can form self-perpetuating amyloid aggregates (termed yeast prions). Yeast prions manifest themselves as epigenetic non-Mendelian heritable elements, transmitted via cytoplasm. They can be detected both microscopically (due to formation of the large aggregated complexes) and phenotypically (due to partial loss of function by the protein in a prion form, that allows for the development of the convenient phenotypic assays). Therefore, yeast model provides unique opportunities for studying de novo amyloid formation, specificity of amyloid transmission, and amyloid clearance.

Our previous research demonstrated induction of prion formation by protein overproduction, crucial role of the chaperone machinery in prion propagation, and connections between the prions, cytoskeletal networks and proteolytic pathways in yeast. In our recent studies, we have explored the effects of heat shock on yeast prions, and have shown that short-term mild heat shock impairs prion propagation by causing the asymmetric prion segregation in cell divisions. We also employ the yeast system for studying the interrelationship between the amyloids and other protein aggregate deposits (for example, aggresomes, peripheral aggregate deposits, and cytoplasmic aggregates of tail-anchored proteins) in the yeast cell.

In addition, we have developed a new model for studying the prion-promoting effects of mammalian amyloidogenic proteins in yeast. This model is based on fusing a mammalian protein to the prion domain of the yeast protein. The resulting chimeric construct is capable of nucleating de novo formation of the yeast prion in the absence of any known pre-existing prions. Thus, amyloidogenic properties of a mammalian protein can be monitored via prion formation by the yeast protein that is easily detectable at the phenotypic level. Moreover, the same features of the mammalian protein important for the amyloid formation in its native environment turn out to be crucial for its amyloid-promoting effect in yeast. Application of this approach to various mammalian proteins, including PrP, will be discussed. 